\title{
Blocking mitochondrial fission-a new treatment approach for Parkinson disease?
}

Impairment of mitochondrial dynamics

-in particular, an imbalance between

mitochondrial fission and fusion-is

emerging as a key pathophysiological

mechanism and a possible therapeutic

target in Parkinson disease (PD).

In a new study published in Nature

Communications, Kim Tieu and colleagues

found that inhibition of the mitochondrial

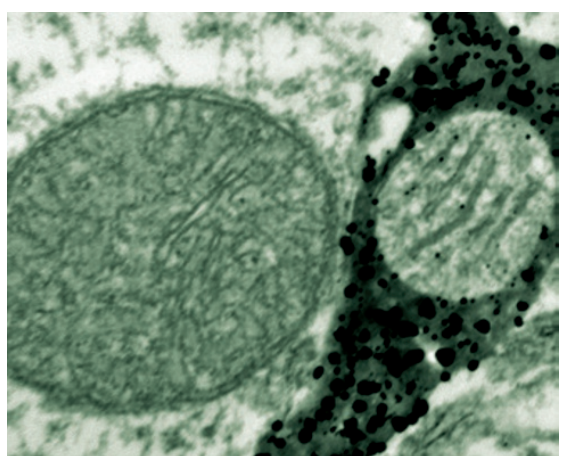

Two mitochondria captured by electron microscopy. One of the mitochondria lies inside a dopaminergic terminal, as indicated by tyrosine hydroxylase immunoreactivity (black spots). Image courtesy of K. Tieu. fission protein dynamin-related protein 1 (Drp1) improved nigrostriatal dopamine release and reduced neurodegeneration in mouse models of PD.

"Previous studies, including our own, reported that blocking of Drp1 function was sufficient to attenuate mitochondrial dysfunction and neurotoxicity in PD cell culture models," explains Tieu. "However, the in vivo significance of this finding needed to be evaluated in animal models of PD."

Tieu's team used two complementary mouse models that recapitulated different aspects of PD: a PINK1 gene deletion model that showed defects in evoked striatal dopamine release, and an MPTP neurotoxic model that exhibited dopaminergic neurodegeneration. Drp1 blockade was accomplished by a smallmolecule inhibitor or by expression of a dominant-negative form of the protein.

In both models, Drp1 blockade promoted mitochondrial fusion and enhanced the release of dopamine from nigrostriatal terminals. In addition, Drp1 inhibition was shown to protect against neurodegeneration in the MPTP model.

"Taken together, our results suggest that Drp1 is a potential therapeutic target for PD," concludes Tieu. "To our knowledge, this is the first demonstration of this nature in animal models."

The researchers plan to test this therapeutic strategy further in genetic and toxin-induced rodent models of $\mathrm{PD}$, and they hope to be able to take the work forward to nonhuman primate models and, ultimately, clinical trials.

\section{Heather Wood}

Original article Rappold, P. M. et al. Drp1 inhibition attenuates neurotoxicity and dopamine release defects in vivo. Nat. Commun. 5, 5244 (2014)

Further reading Cui, M. et al. Perturbations in mitochondrial dynamics induced by human mutant PINK1 can be rescued by the mitochondrial division inhibitor mdivi-1. J. Biol. Chem. 285, 11740-11752 (2010) 